\title{
Study on Motion Recognition Scheme Using Acceleration Sensor in Mobile Devices
}

\author{
Younghoon Kim, Sangyub Kim, Dongmin Kang, Hwangsoo Park, Nammoon Kim, \\ Sung Hyun Yang and Youngok Kim \\ Department of Electronic Engineering, Kwangwoon University, 447-1 Wol gye-dong, \\ No won-gu, Seoul, 139-701, Republic of Korea \\ kimyoungok@kw.ac.kr
}

\begin{abstract}
In this paper, a motion recognition scheme using acceleration sensor of smart devices is proposed and is experimentally analyzed with a server. Sensor data of 3-axis acceleration of a smart device is collected and 2nd order Butterworth low pass filter (LPF) is applied to reduce noise. With the collected sensor data, the various motions such as falling, sitting, lying and walking are distinguished one another. Based on the fixed threshold by the probability approach, falling motion is especially distinguished from other motions as an indication of emergency situation. A server displays current states and alarm states. The proposed scheme is evaluated through experiments. The results of experiments show that the accuracy of motion recognition is more than $94 \%$ in all the motions. As for a falling recognition, the accuracy is $96 \%$.
\end{abstract}

Keywords: Motion recognition, Mobile device, Acceleration sensor, Butterworth, Low pass filter

\section{Introduction}

In these days, the important of health care system has been increased. Especially, a large number of elderly, children, and patient get in trouble with falling and they get serious injuries because of the unnoticed situation after falling. According to the national health insurance corporation, the ratio of person who experiences falling accident in a year is 28 35\% after 65 years old [1,2]. Moreover, it is increased to $35 \%$ after 70 years old and 32 42\% after 75 years old. Furthermore, it is up to 50\% after 80 years old. The elderly person who has fallen needs to be taken care quickly because it can lead to big threat $[3,4]$. For example, the ratio of death for the fallen elderly person is twice time more than that of the un-fallen elderly person. In other case, patients who need medical care in the ordinary life are always cared by hospital, but it has limiting factors such as medical equipment and the number of people who can care patients. Also, existing equipment have to set up in various parts of body and it makes patients to act an awkward movements [5, 6]. Therefore, they need to have health care management by a care system and by solving existing limiting factors. The objective of our proposed scheme is to manage patients more conveniently and quickly by using general smart phones. For development of system that can be applied to actual situation, various situations and conditions are considered and various motions such as falling, sitting, lying and walking also are considered. 


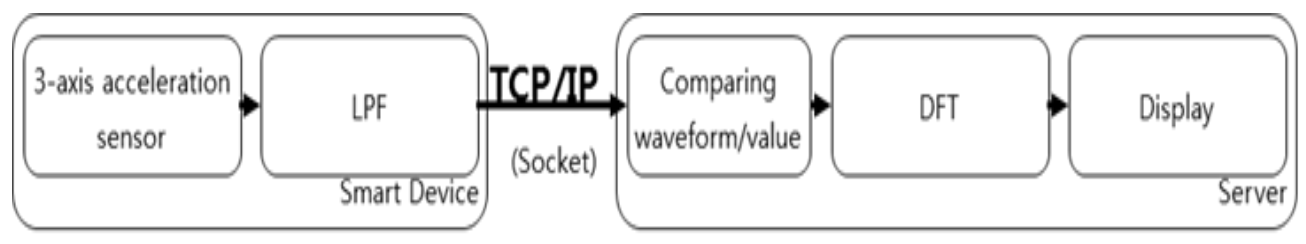

Figure 1. System Block Diagram

The difference between existing feature phone and smart phone is that the smart phone includes acceleration sensor. This sensor can be used to detect motion of users. Currently, penetration rate of smart phone is dramatically increased. Also, technology of sensor will continuously be developed and many applications will be developed by using the technology [7-10]. Many people sometimes forget to equip their medical equipment, but they usually do not forget to bring their smart phone. If their smart phone has fall detection scheme, their falling event can be checked by medical officer in real-time while other motions, such as sitting, lying and walking, are also detected if it is required. For these reasons, a motion recognition scheme adapted to a smart phone is proposed in this paper.

The rest of the paper is organized as follows. In Section 2, the proposed system is briefly described. In Section 3, the proposed scheme is evaluated with experiments and its performance is reviewed. Finally, our concluded remarks including the extended suggestions and future works are summarized in Section 4.

\section{System Description}

Figure 1 shows a simple block diagram of proposed system. As shown in the figure, the data of acceleration sensor is collected from the device and the effect of noise is reduced by using the low pass filter (LPF) for decreasing detection error rates. For more detailed process, Figure 2 shows a flow chart of proposed scheme. As shown in the figure, each motion is distinguished with different threshold. If acceleration value exceeds a falling threshold and waiting time goes over a pre-determined period, the system determines falling event. Also, the device informs to a server about emergency situation through TCP/IP communication scheme. The server displays the current status and informs alert. If acceleration value does not exceed a threshold or waiting time does not go over a pre-determined period, the system calculates the error power and the sum of DFT samples, so it can distinguish sitting and lying motions. If falling, sitting, and lying motions are not determined, and the sum of DFT samples is more than 23 , it recognizes as walking motion.

\subsection{Three-axis Acceleration Sensor}

There are various sensors that can detect movement of a user: gyroscope, geomagnetic sensor, accelerometer, and gravity sensor. Among them, accelerometer is a sensor to recognize change of acceleration and it can also measure vibration and impulse. Since the accelerometer can recognize movement of object delicately, it is used in wide fields. Moreover, the each axis value of acceleration sensor is affected by moving and position of device. Therefore, the 3 -axis acceleration sensor is used for the proposed scheme. 


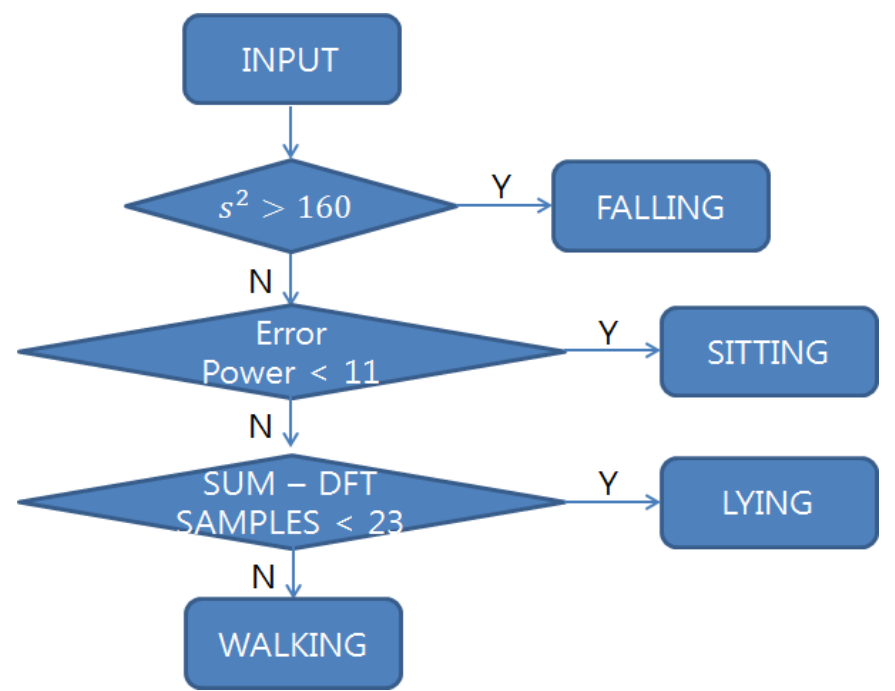

Figure 2. Flow Chart of Proposed Scheme

\subsection{Socket Communication Technology}

The communication technology to transfer data can be classified into two categories: Socket and HTTP. There are two ways using socket: Protocol/ Internet Protocol (UDP/IP) and Transmission Control Protocol/ Internet Protocol (TCP/IP). TCP requests repeated data to destination, if there are some problems in the transmission. This feature is different with UDP, so it has reliability about transmission of data. Also, TCP is generally used in the long distance telecommunication and it is connection oriented protocol, so it takes long time relatively. Figure 3 shows an outline of socket communication.

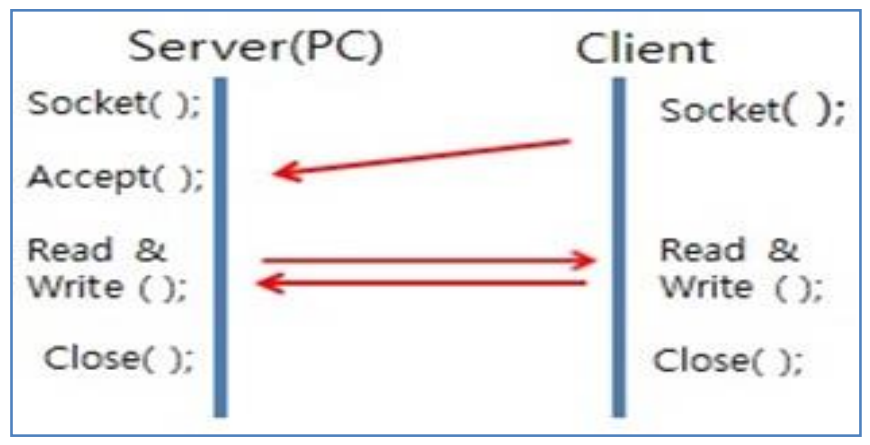

Figure 3. Outline of Socket Communications

\section{Experimental Results}

A smart phone, which is named IM-A690S and is based on android OS 2.3.3 (Gingerbread), is used for the experiments. The smart phone has a 3.5" WVGA, 1G internal memory and 512 RAM. The server is composed through the eclipse software. The height of experimenter is $162 \mathrm{~cm}$. The results of experiment are affected by the height of experimenter because the changing amount of acceleration sensor is different according to the height. In this experiment, therefore, the height of experimenter is 
fixed. Sampling period for getting sensing data is $50 \mathrm{~ms}$. 80 samples were collected as 1 count to detect current states for 4 seconds.

\subsection{Recognizing Falling Motion}

The each axis value of acceleration sensor is affected by moving and position of device. In order not to be affected by rotation and position of the device, square sum of each axis value, $S^{2}$, is defined as follow:

$$
S^{2}=x^{2}+y^{2}+z^{2},
$$

where $\mathrm{x}, \mathrm{y}, \mathrm{z}$ are sensing values from each axis.

Since the falling motion can give an affect for 3-direction $\mathrm{x}, \mathrm{y}, \mathrm{z}$., the system distinguishes the falling motion from walking, sitting, and lying motions by using the $S^{2}$.

Figure 3 shows the distribution of $S^{2}$ values for various motions. As show in the figure, the minimum value of falling motion is 214 and maximum value of the other motions is 113 . Therefore, we set the threshold on 160, which is between 214 and 113 with margin. However, other motions cannot be recognized with $S^{2}$, because $S^{2}$ is overlapped at same regions, so we have to find another method to distinguish among motions.

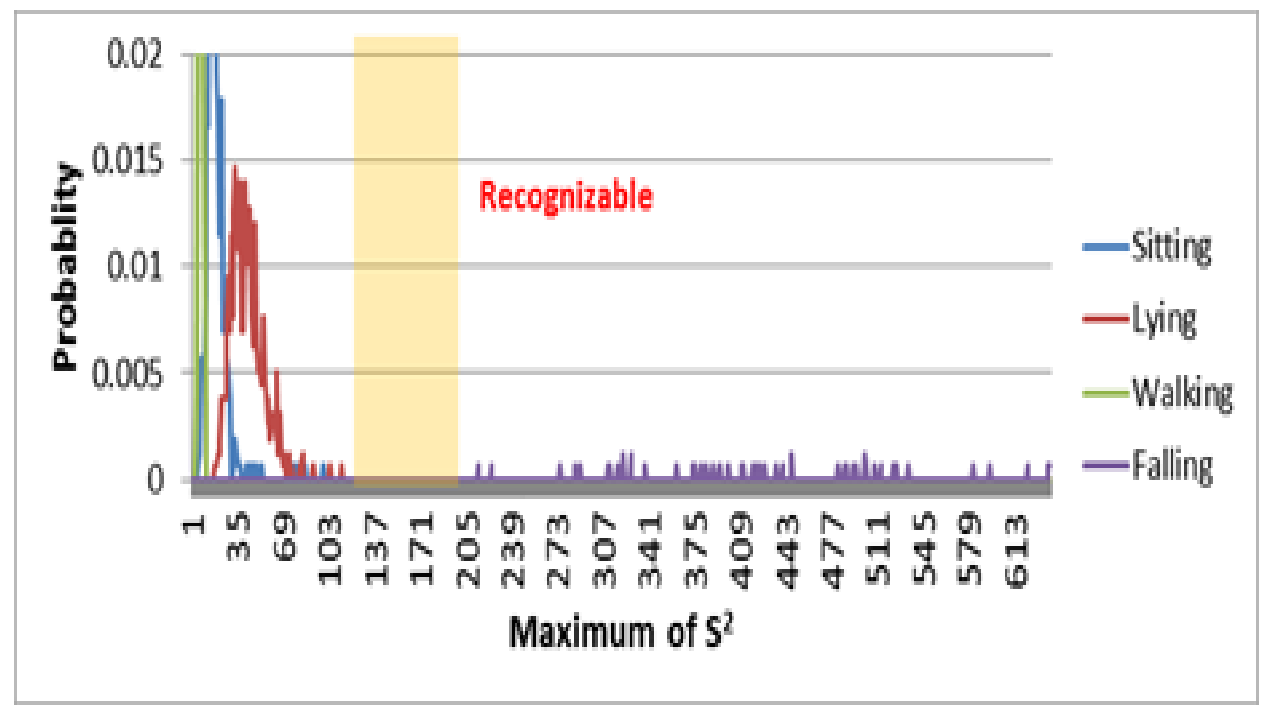

Figure 4. Distribution of $S^{2}$ Values for Various Motions
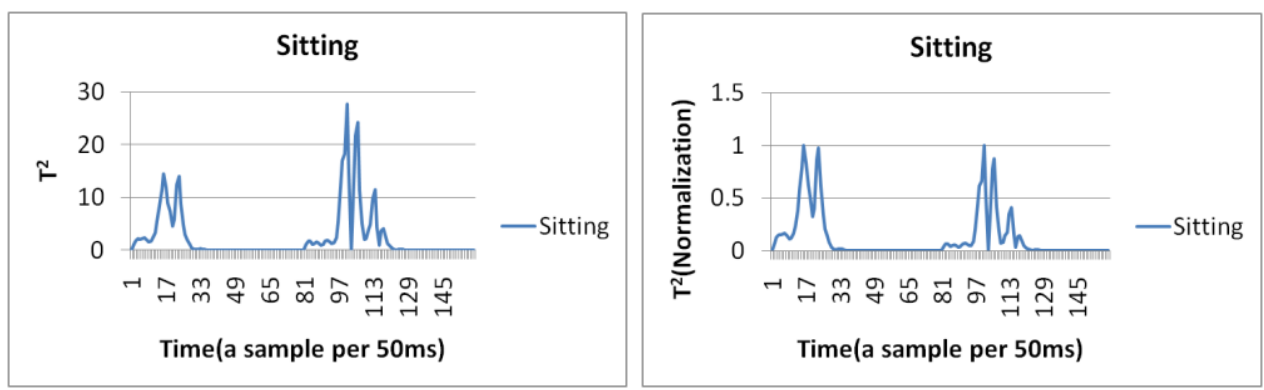

Figure 5. Distribution of $T^{2}$ of Sitting and its Normalized Data 

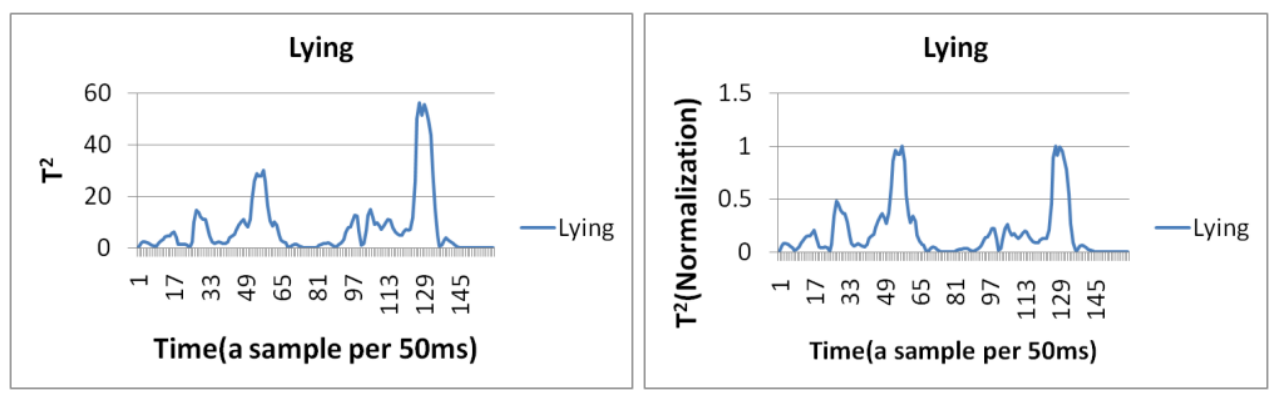

Figure 6. Distribution of $T^{2}$ of Lying and its Normalized Data
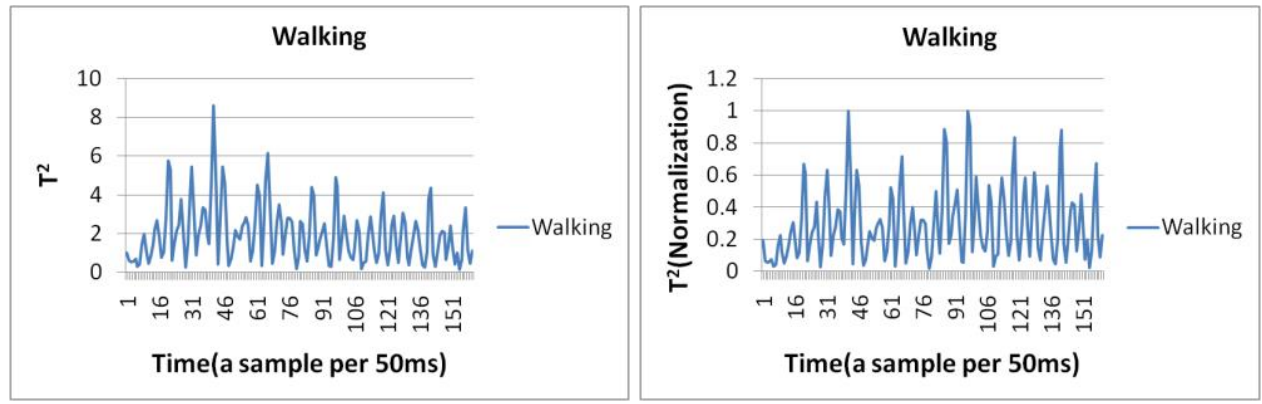

Figure 7. Distribution of $T^{2}$ of Walking and its Normalized Data

\subsection{Recognizing Sitting Motion}

To distinguish sitting, lying, and walking motions, we move to only y and z direction after recognizing falling motion. Therefore, square sum of each axis value, $T^{2}$, is defined as follow:

$$
T^{2}=y^{2}+z^{2}
$$

We determine the motion with the interval of 80 samples, which is for 1 count. Figures 5, 6, 7 show the distribution of $T^{2}$ values for 2 counts for various motions. As show in the figures, the changes of sitting data happens at heading points of intervals, while changing data for other motions, lying and walking, exist relatively longer interval than sitting motion. Therefore, we assume that the sitting motion can be distinguished from other motions with post-processed 80 samples, which is called error power. The error power is the sum of absolute value of 80 samples, which is obtained by subtracting averaged values for 80 samples that is obtained by averaging 512 counts of sitting as shown in Figure 8 to the current 80 samples. Before this process, it needs to normalize to maximum value of each interval because quantity of change different whenever it is measured as shown in the figures. If the error power is less than 10 , it can be recognized as the sitting motion. Otherwise, it determines the motion as lying or walking.

Figure 9 shows the distribution of error power for sitting, lying, and walking motions. As shown in the figure, we can divide Sitting-Sitting and Sitting-other motions, but the SittingSitting and Sitting-Lying are overlapped around 11th error power. According to measured data, however, we missed to determine 3 counts of 513 sitting counts, and the miss rate was almost $0 \%$. 


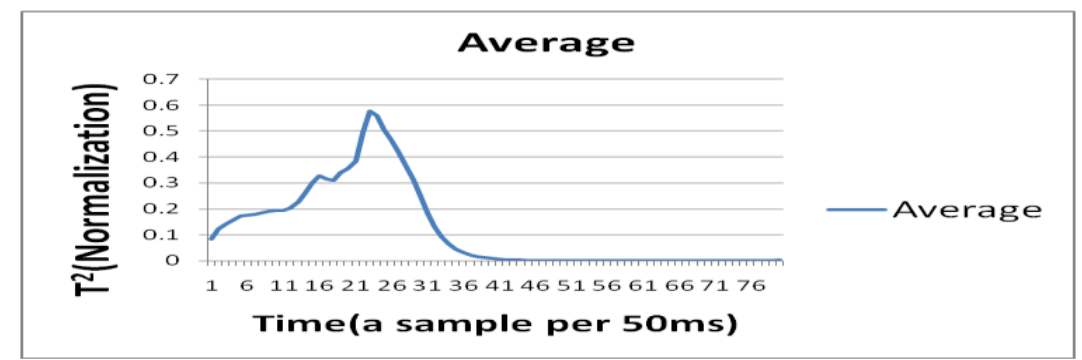

Figure 8. Averaged 80 Samples of Sitting Data (Total 512 Counts)

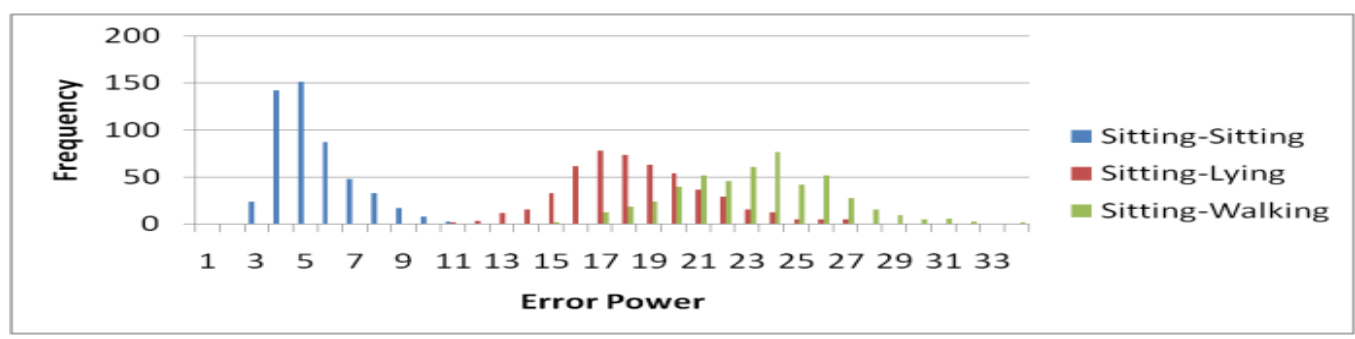

Figure 9. Distribution of Error Powers

\subsection{Recognizing Lying \& Walking Motion}

It remains to recognize lying motion and walking motion. At frequency analysis, we notice that the sum of 23 28th samples, which represents $3.6 \sim 4.4 \mathrm{~Hz}$, is less than 23 for lying motion. Meanwhile, when the sum is greater than 23, it can be identified to walking motion and the error percent of this case was also $0 \%$. Figure 10 shows the example of frequency analysis of 20 counts for lying and walking motions. Note that the max/min values of each motion are as follows:

- Maximum value of sum of lying: 18

- Minimum value of sum of walking: 28

Figure 11 shows the sum of DFT of samples. As shown in the figure, we can recognize lying and walking motions. Note that we marked recognizable band in the figure.

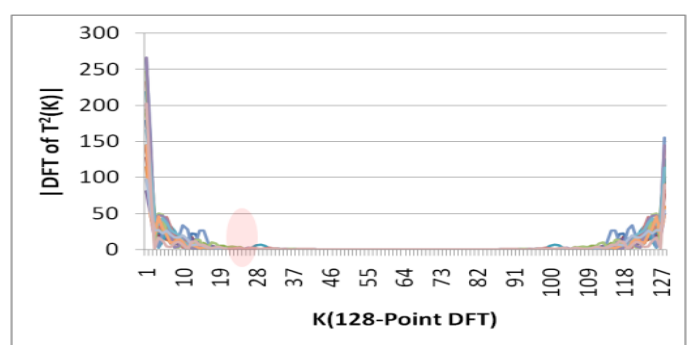

(a)

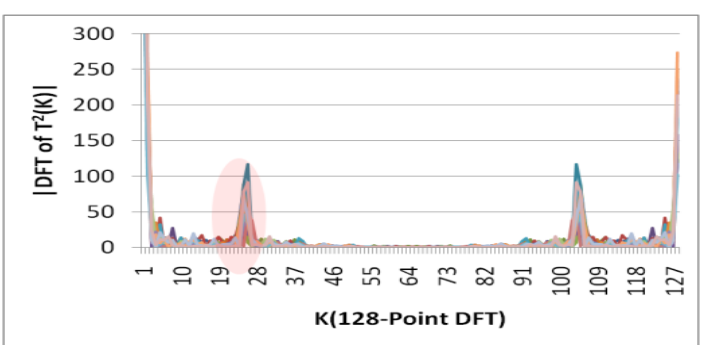

(b)

Figure 10. Frequency Analysis of $T^{2}$ (norm) of (a) Lying (20counts) and (b) Walking (20counts) 


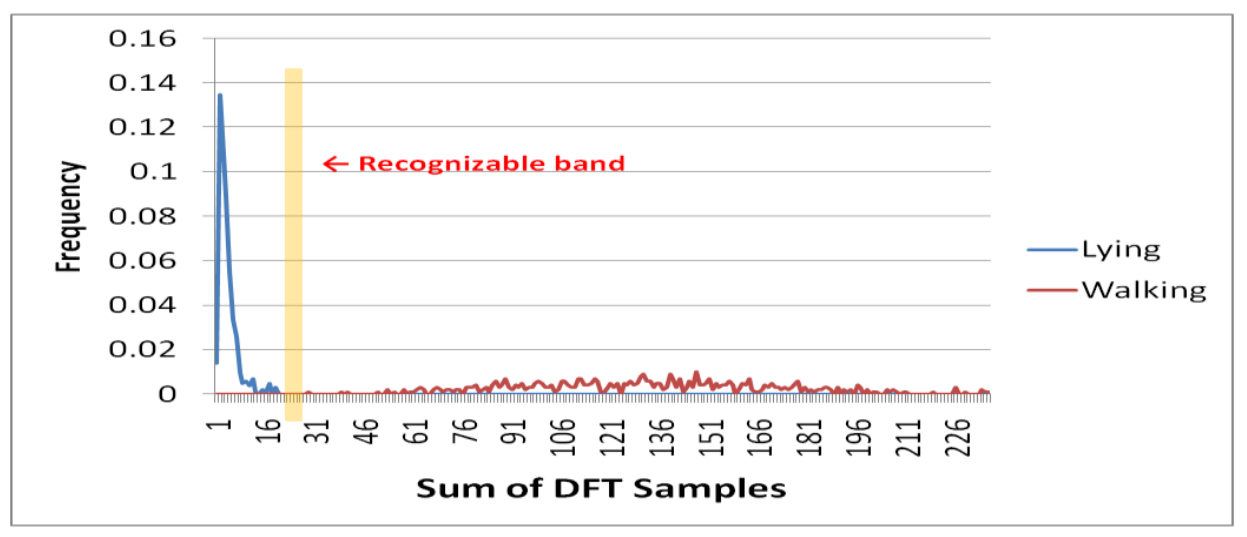

Figure 11. Frequency of Sum of DFT Samples: Lying and Walking

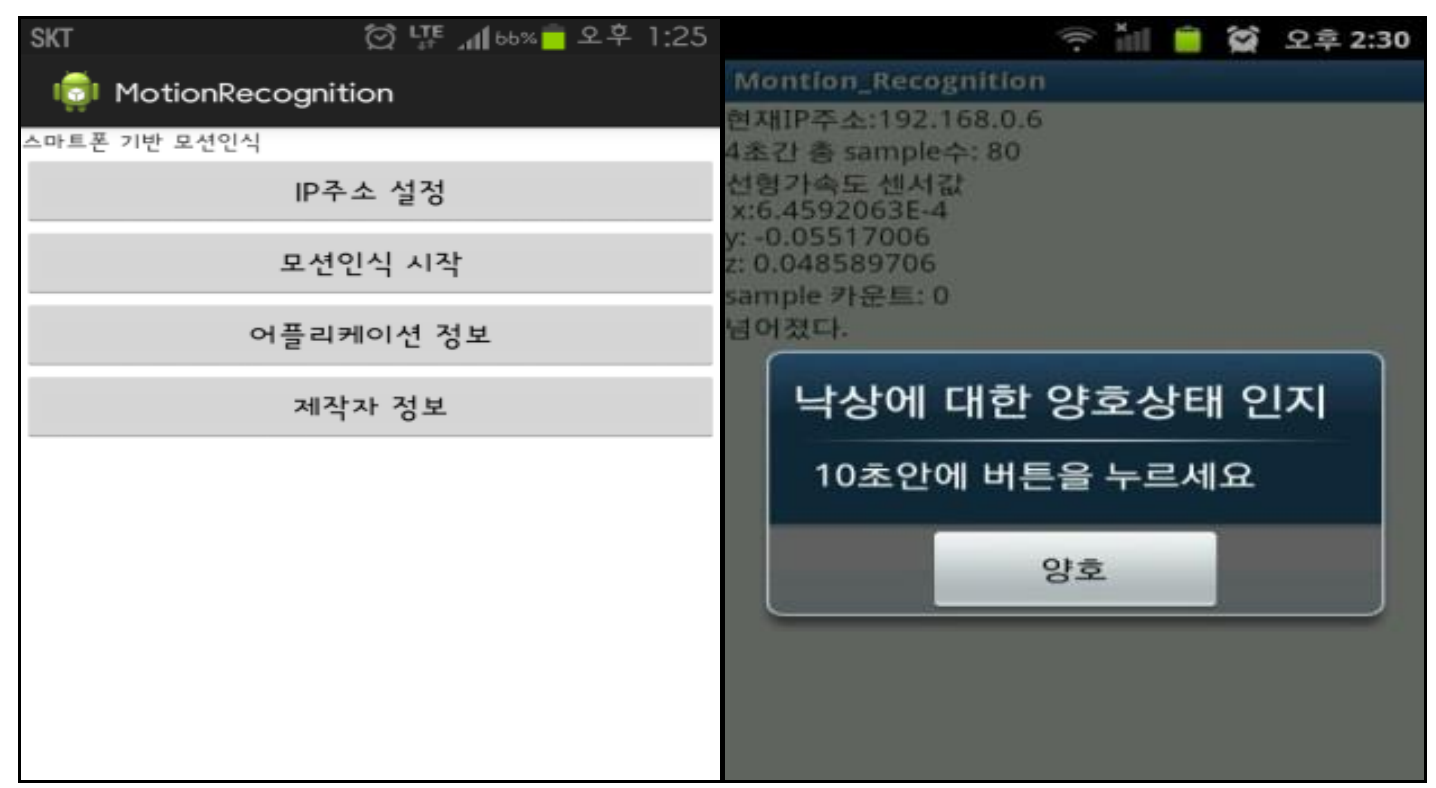

Figure 12. Display of Mobile Device

Table 1. Result of Experiments (Units: Times)

\begin{tabular}{|c|c|c|c|c|}
\hline & Falling & Sitting & Lying & Walking \\
\hline Success & 96 & 190 & 192 & 188 \\
\hline Failure & 4 & 18 & 10 & 14 \\
\hline Total & 100 & 200 & 200 & 200 \\
\hline
\end{tabular}

\subsection{Summary of Performance Evaluation}

Figure 12 depicts display on the mobile device. Left figure shows the menu for IP address setup, motion recognition implement and etc. Right figure shows the sensing data and current acceleration values. If falling is detected, the device opens a popup window to protect wrong recognition and it sends the measured data to the server. 
Experiment is performed 100 times for falling motion, 200 times for the other motions. Table 1 shows the results of experiment. As shown in the table, the falling motion was successfully recognized 96 times, while sitting was 190 times, lying was 192 times, and walking was 188 times.

\section{Conclusions}

In this paper, a motion recognition scheme using acceleration sensor of smart devices is proposed and is experimentally analyzed with a server. We developed the system to recognize various motions such as falling, sitting, lying, and walking. The results of experiment show the accuracy of $96 \%$ in falling recognition and the sitting is $95 \%$, the lying is $96 \%$, and the walking is $94 \%$. Beyond the falling detection in the limited initial statement as standing, the proposed scheme extends to more initial statements like walking, so it can offer more accurate detection of falling event.

During the work, there were some limitations. First, it is hard to recognize combination motion with rotation (included $\mathrm{x}$-axis) because acceleration value of $\mathrm{x}$-axis is not changed significantly. Second, during the process to recognize walking motion, the interval of sum can be changed as walking speed varies.

For development of the scheme recognizing various motions, it needs to be considered the height of people because the height is the biggest causes to consider threshold that recognize the motion. Therefore, various situations and conditions will be considered as future works.

\section{Acknowledgements}

This work was supported by the Industrial Strategic technology development program (10041788, Development of Smart Home Service based on Advanced Context-Awareness) funded By the Ministry of Trade, industry \& Energy (MI, Korea).

\section{References}

[1] Z. Meng and Q. Hongwu, "The Gain Analysis about the Elder Fall Risk", Fifth International Conference on Intelligent Networks and Intellingent Systems (ICINIS), Tianjin, China, (2012) November 1-3.

[2] M. C. Nevitt, S. R. Cummings, S. Kidd and D. Black, "Risk factors for recurrent nonsyncopal falls", A prospective study, Journal of the American Medical Association, vol. 261, (1989).

[3] G. Salkeld G, I. D. Cameron, R. G. Cumming, S. Easter, J. Seymour, S. E. Kurrle and S. Quine, "Quality of life related to fear of falling and hip fracture in older women: a time trade off study", British Medical Journal, vol. 320, (2000).

[4] R. G. Cumming, G. Salkeld, M. Thomas and G. Szonyi, "Prospective study of the impact of fear of falling on activities of daily living, SF-36 scores, and nursing home admission”, Journals of Gerontology, Series A, Biological Sciences and Medical Sciences, vol. 55, (2000).

[5] K. Brewer, C. Ciolek and M. F. Delaune, "Falls in community dwelling older adults: Introduction to the problem", APTA Continuing Education Series, (2007), pp. 38-46.

[6] S. Brownsell and M. Hawley, "Automatic fall detectors and the fear of falling", Journal of Telemedicine and Telecare, vol. 10, no. 5, (2004).

[7] A. Anjum and M. U. Ilyas, "Activity recognition using smartphone sensors", IEEE Consumer Commun. and Networking Conference (CCNC), Nevada, USA, (2013) January 11-14.

[8] O. Yurur, C. H. Liu and W. Moreno, "Unsupervised posture detection by smart phone accelerometer", Electronics Letters, vol. 49, no. 8, (2013).

[9] H. Byun and J. Lee, International Journal of Smart Home, vol. 6, no. 3, (2012).

[10] J. Lee, Y. Chuah and K. Chieng, International Journal of Smart Home, vol. 7, no. 3, (2013). 